\title{
Power System Stabilizer Design for Multi-machine Power System Using Genetic Algorithm
}

\author{
Abinet Tesfaye \\ School of Electrical and \\ Electronic Engineering, North \\ China Electric Power \\ University, and \\ Goldwind and Etechwin \\ Electric Co., Ltd \\ Beijing, China \\ J. H. Zhang \\ School of Electrical and \\ Electronic Engineering, North \\ China Electric Power \\ University \\ Beijing, China
}

\author{
Dereje Shiferaw \\ Department of Electrical and \\ Computer Engineering, Adama \\ Science and Technology \\ University \\ Adama, Ethiopia
}

\author{
D. H. Zheng \\ Goldwind and Etechwin \\ Electric Co., Ltd \\ Beijing, China
}

\begin{abstract}
This paper uses genetic algorithm (GA) framework integrated with the classical Lyapunov's parameter optimization method employing an Integral of Squared Error (ISE) criterion to optimally tune the parameters of the power system stabilizers (PSSs) for a multi-machine system, consisting of three machines and ten interconnected buses, which is taken from the national electric power grid of Ethiopia. The issue of optimally tuning the parameters of the PSS is converted into an optimization problem that is solved via the GA algorithm. Within the GA process, a potential solution - the PSS parameter setting - is coded as an individual, which is part of a population of such potential solutions randomly generated, and by applying the survival of the fitness principle based on each individual's fitness with respect to the objective, a sound basis for finding the best individual, i.e. global optimum solution, is created. Simulation results are presented to show the effectiveness of the proposed approach, for various system loading conditions and other disturbances such as perturbation in mechanical torque inputs to the machines, and have been performed with satisfactory results with the design and integration of PSS to the power system investigated in this paper.
\end{abstract}

Keywords: Dynamic stability, electromechanical oscillations, genetic algorithm (GA), Integral of Squared Error (ISE), Lyapunov equation, power systems, power system stabilizers (PSSs).

\section{INTRODUCTION}

PSSs are auxiliary control devices on synchronous generators, used in conjunction with their excitation systems to provide control signals toward enhancing the system damping of electromechanical oscillations and extending power transfer limits, thus maintaining reliable operation of interconnected power systems.

Up to now conventional PSSs (CPSSs) are used in the industry because of their simplicity and their relatively good performance around the nominal operating point (Rogers, 2000).

However, conventional PSS based on a single operating condition cannot maintain adequate system stability over a wide range of operating conditions. In many instances, inadequate tuning procedures of PSS based on the sequential design have led to the destabilization of the entire system (Rogers, 2000). There is a need to find a systematic tuning procedure of PSS so as to achieve optimum parameter settings over a wide range of operating conditions [16].

Genetic algorithm based applications to tune the parameters of PSS have been reported in [5]-[8]. A GA based optimization method has been used in [6] to tune the parameters of a rulebased PSS; this way, the advantages of the rule-based PSS such as its robustness, less computational burden and ease of realization are maintained. Introduction of GAs helps obtain an optimal tuning for all PSS parameters simultaneously, which thereby takes care of interactions between different PSS [21].

In [7], simultaneous tuning for all the PSS in the system using a GA based approach has been developed. The GA seeks to shift all eigenvalues of the system within a region in the stable domain. In [8], a multiobjective design of PSSs in a multimachine power system operating at various loading conditions and system configurations is achieved using a GA search process. A multiobjective problem is formulated to optimize a 
composite set of objective functions comprising the damping factor, and the damping ratio of the lightly damped electromechanical modes, and the effectiveness of the suggested technique is confirmed through eigenvalue analysis and nonlinear simulation results.

Zhang and Coonick [5] proposed a GA based computational procedure to select PSS parameters simultaneously in multimachine power systems, by solving a set of inequalities that represent the objectives of optimization problem.

In [8], a GA based tuning technique of fixed structure damping controllers over a prespecified set of operating conditions is proposed and demonstrated for large-scale realistic systems. It is emphasized here the importance of an accurate fitness function and the fact that a power system expert's input in the designing stage of the optimization process is very important, and sometimes required to select the best solution out of a pool of solutions resulted from the algorithm [24].

The proposed method, in this research paper, integrates the classical parameter optimization approach, involving the solution of Lyapunov equation, within a genetic search process. The method ensures that for any operating condition within a pre-defined domain, the system remains stable when subjected to small perturbations. The optimization criterion employs a quadratic performance index that measures the quality of system dynamic response within the tuning process. The solution thus obtained is globally optimal and robust. The proposed method has been tested on the lead-lag type PSS structure. System dynamic performances with PSS tuned using the proposed technique are satisfactory for different load conditions and system configurations both for the nominal and off-nominal operating conditions.

The paper is organized as follows: Section 2 introduces the dynamic stability model of the power system. Section 3 describes an overview of genetic algorithm. Designing the PSSs and optimal tuning of the parameters is described in section 4. Eigenvalue analysis and time domain simulations are provided in section 5, and the paper is concluded in section 6.

\section{SYSTEM MODEL}

The system considered in this paper is a three machine ten-bus power system taken from the national electric power grid of Ethiopia. It comprises of three synchronous generators (MEWA, AWASH2, and KOKA) which are interconnected together through a distribution and transmission network of ten buses in a ring network topology as shown in Figure 1.

Each machine is represented by the two-axis model (d-q axis). The machines are equipped with a simple AVR (Yu, 1983). The dynamics of the system are described by a set of nonlinear differential equations. However, for the purpose of controller design, these equations are linearized around the nominal operating conditions [16].

The transfer function block diagram in Figure 2 describes the dynamics of the $\mathrm{i}^{\text {th }}$ machine in a multi-machine power system [10], where $\Delta \omega_{\mathrm{i}}$ is the angular speed deviation, $\Delta \delta_{\mathrm{i}}$ is rotor angle deviation, $\Delta \mathrm{E}_{\mathrm{qi}}{ }_{\mathrm{i}}$ is $\mathrm{q}$-axis transient air-gap voltage deviation, $\Delta \mathrm{E}_{\mathrm{fdi}}$ is excitation voltage deviation, $\Delta \mathrm{T}_{\mathrm{mi}}$ is mechanical torque deviation, $\Delta \mathrm{T}_{\mathrm{ei}}$ is electrical torque deviation, $\mathrm{T}^{\prime}{ }_{\text {doi }}$ is $\mathrm{d}$-axis transient time constant, $\mathrm{K}_{\mathrm{Ai}}$ is excitation system gain, $T_{\mathrm{Ai}}$ is excitation system time constant, $M_{i}$ is inertia constant of the $i^{\text {th }}$ machine.
This is a generalization of the extensively used single machine connected to its terminal bus or infinite bus transfer function block diagram [2] and takes into account the interaction between machines via $\mathbf{K}$ matrices, which are square matrices with order equal to the number of generators $\left(\mathrm{n}_{\mathrm{g}}\right)$.

These $\mathbf{K}$ matrices are derived from the electric torque expression (K1, K2), field winding circuit equation (K3, $\mathrm{K} 4$ ), and from the terminal voltage magnitude (K5, K6), and the diagonal elements of the $\mathbf{K 1}, \mathbf{K} 2, \ldots, \mathbf{K} \mathbf{6}$ matrices determine the machine's dynamics, while the off-diagonal elements model the dynamic interactions between machines. Observe that in this block diagram the PSS is not represented, for convenience [19].

The number of state variables is $n_{v} x n_{g}$, where $n_{v}$ is the number of state variables used to model one machine and its excitation system.

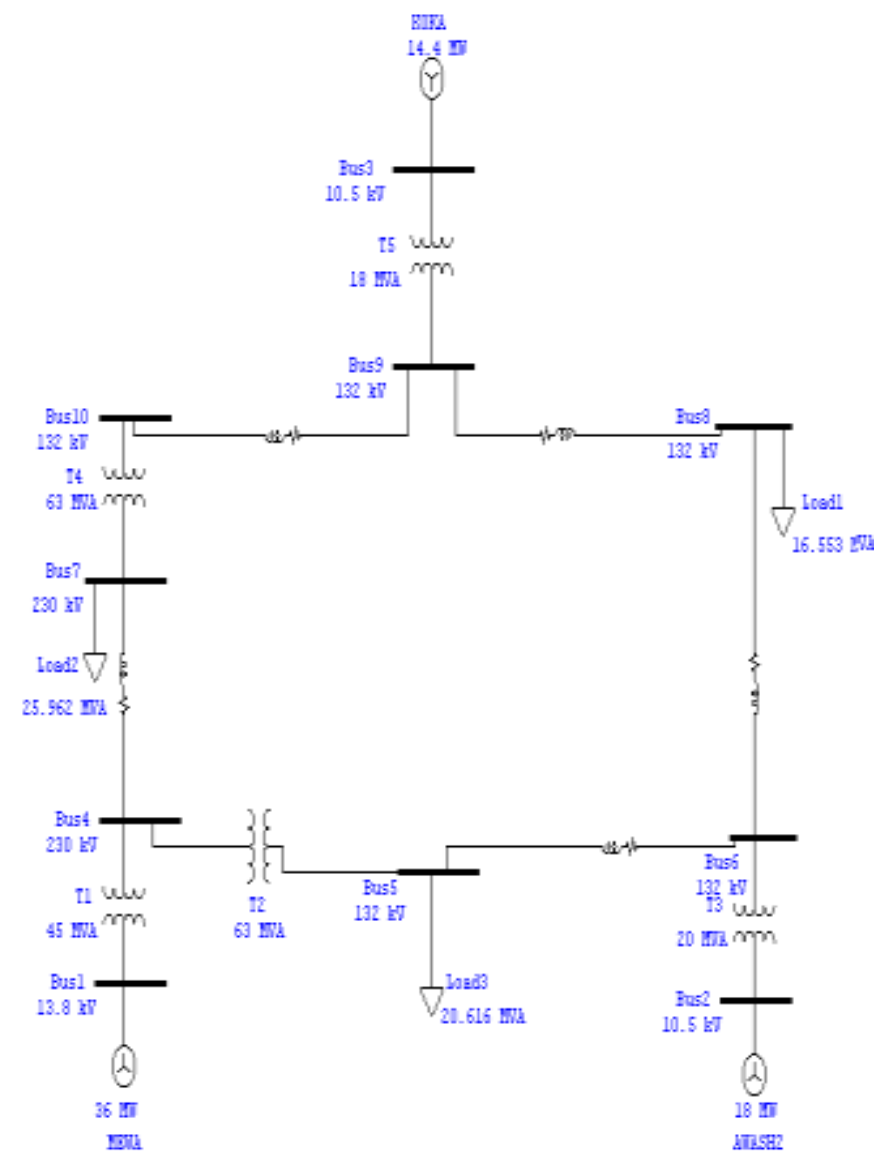

Figure 1. Three-machine ten-bus power system (Source: Ethiopian National Electric Power Grid)

Based on the transfer function block diagram (Figure 2), the system dynamics can be expressed by a set of linear differential equations in the small-perturbation variables $\Delta \omega \mathrm{i}$, $\Delta \delta_{\mathrm{i}}, \Delta \mathrm{E}_{\mathrm{q}}{ }_{\mathrm{i}}, \Delta \mathrm{E}_{\mathrm{fdi}}$ as follows:

$$
\begin{aligned}
& \frac{d}{d t}\left(\Delta \omega_{i}\right)=\frac{D_{i}}{M_{i}} \Delta \omega_{i}-\frac{K_{1 i}}{M_{i}} \Delta \delta_{i}-\frac{K_{2 i}}{M_{i}} \Delta E_{q i}^{\prime}+\frac{1}{M_{i}} \Delta T_{m i} \\
& \frac{d}{d t}\left(\Delta \delta_{i}\right)=2 \pi f \Delta \omega_{i} \\
& \frac{d}{d t}\left(\Delta E_{q i}^{\prime}\right)=-\frac{K_{4 i}}{T_{d o i}^{\prime}} \Delta \delta_{i}-\frac{1}{T_{d o i}^{\prime} K_{3 i}} \Delta E_{q i}^{\prime}+\frac{1}{T_{d o i}^{\prime}} \Delta E_{f d i} \\
& \frac{d}{d t}\left(\Delta E_{f d i}\right)=-\frac{K_{A i} K_{5 i}}{T_{A i}} \Delta \delta_{i}-\frac{K_{A i} K_{5 i}}{T_{A i}} \Delta E_{q i}^{\prime}-\frac{1}{T_{A i}} \Delta E_{f d i}+\frac{K_{A i}}{T_{A i}} \Delta u_{E i}
\end{aligned}
$$




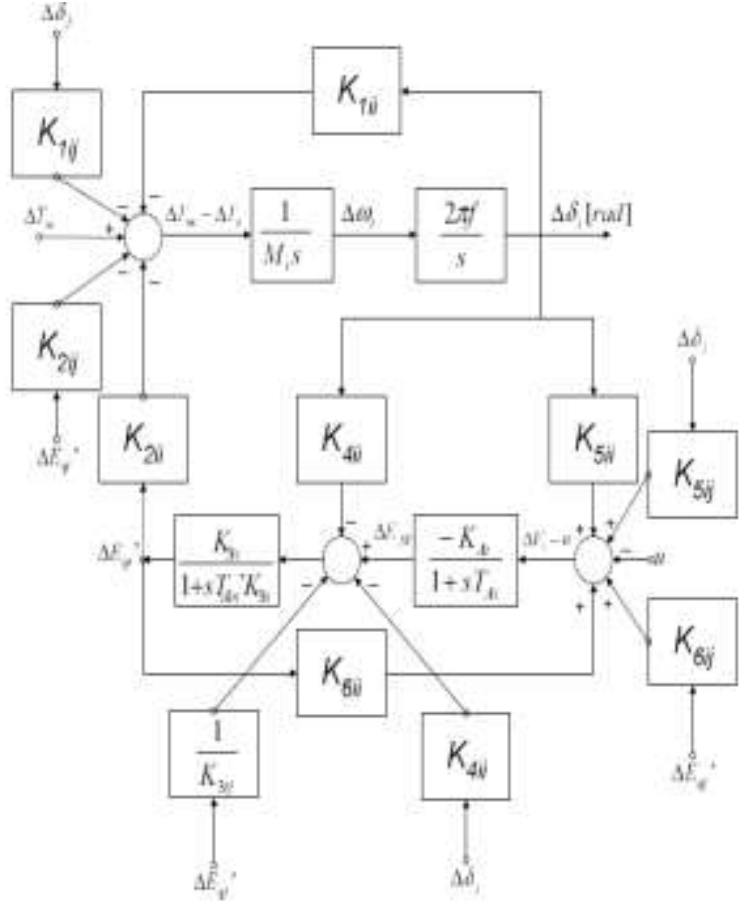

Figure 2. Transfer function block diagram representation of a multi-machine system for small-signal stability analysis

Using vector-matrix notation and the above set of equations (1), the state-space model for the three-machine system is expressed as follows:

$$
\frac{d}{d t} \underline{X}(t)=\mathrm{A} \cdot \underline{X}(t)+\mathrm{B} \cdot \underline{U}(t)+\Gamma \cdot \underline{p}(t)
$$

Where $\underline{X}(t), \underline{U}(t)$ and $\underline{p}(t)$ are state, control and perturbation vectors, respectively and they are expressed as follows:

$\underline{X}(\mathrm{t})=\left[\Delta \omega_{1}(\mathrm{t}) \Delta \delta_{1}(\mathrm{t}) \Delta \mathrm{E}^{\prime} \mathrm{q}_{1}(\mathrm{t}) \Delta \mathrm{Efd}_{1}(\mathrm{t}) \ldots\right.$

$$
\begin{aligned}
& \Delta \omega_{2}(\mathrm{t}) \Delta \delta_{2}(\mathrm{t}) \Delta \mathrm{E}^{\prime} \mathrm{q}_{2}(\mathrm{t}) \Delta \mathrm{Efd}_{2}(\mathrm{t}) \ldots \\
& \left.\Delta \omega_{3}(\mathrm{t}) \Delta \delta_{3}(\mathrm{t}) \Delta \mathrm{E}^{\prime} \mathrm{q}_{3}(\mathrm{t}) \Delta \mathrm{Efd}_{3}(\mathrm{t})\right]^{\mathrm{T}}
\end{aligned}
$$

$\underline{\mathrm{U}}(\mathrm{t})=\left[\begin{array}{lll}\Delta \mathrm{u}_{1}(\mathrm{t}) & \Delta \mathrm{u}_{2}(\mathrm{t}) & \Delta \mathrm{u}_{3}(\mathrm{t})\end{array}\right]^{\mathrm{T}}$

$\mathrm{p}(\mathrm{t})=\left[\begin{array}{lll}\Delta \mathrm{T}_{\mathrm{m} 1} & \Delta \mathrm{T}_{\mathrm{m} 2} & \Delta \mathrm{T}_{\mathrm{m} 3}\end{array}\right]^{\mathrm{T}}$

$\mathbf{A}, \mathbf{B}$ and $\boldsymbol{\Gamma}$ are the state, control and perturbation matrices, respectively.

\section{OVERVIEW OF GENETIC ALGORITHM}

Genetic Algorithms are global search techniques providing a powerful tool for optimization problems by miming the mechanisms of natural selection and genetics. These operate on a population of potential solutions applying the principle of survival of the fittest to produce better and better approximations to a solution. In each generation, a new set of approximations is created by selecting the individuals according to their level of fitness in the problem domain and breeding them together using operators borrowed from natural genetics [11]. Thus, the population of solutions is successively improved with respect to the search objective by replacing least fit individuals with new ones (offspring of individuals from the previous generation), better suited to the environment, just as in natural evolution [20].
According to Goldberg [11] and [25], GAs are different from other optimization and search procedures in four ways:

a. GA work with a coding of the parameter set, not the parameters themselves.

b. GA search from a population of points, not a single point.

c. GA use payoff information, not derivatives or other auxiliary knowledge.

d. GA use probabilistic transition rules, not deterministic rules.

Figure 3 shows a flowchart diagram of genetic algorithm.

The process commences with random generation of a pool of possible solutions, i.e. the population and the individuals that form it. Each individual in the population, also called chromosome is represented by a string, which is formed by a number of sub-strings equal to the number of the problem's variables. Each variable is coded in a suitable coding system (binary, integer, real-valued, etc). The population size and the chromosome size are kept constant during the whole search process.

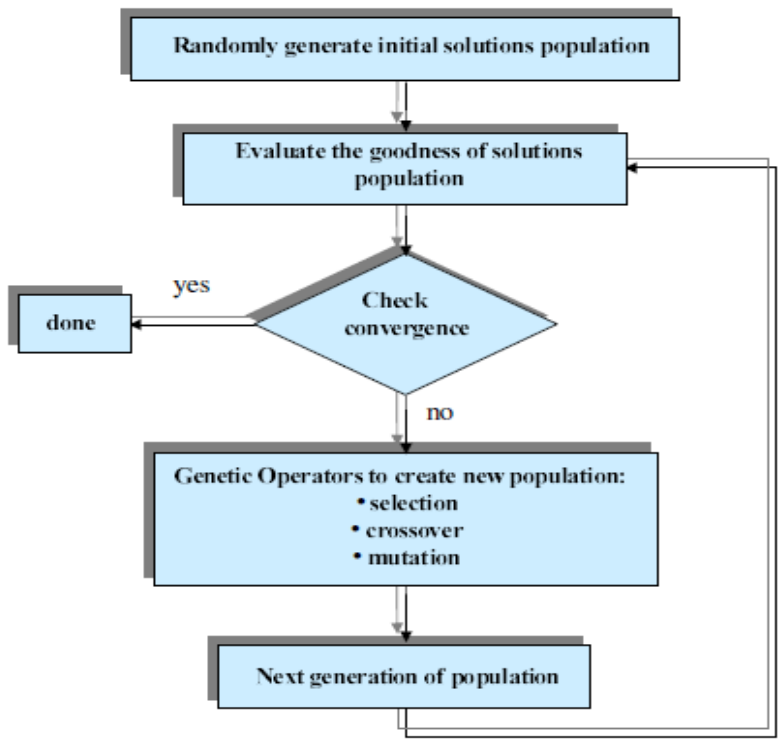

Figure 3. Genetic algorithm flowchart diagram

\section{DESIGN OF THE PSS}

The main goal of this study is to optimize the parameters of the PSSs such that controllers simultaneously stabilize a family of system models. It was found that a double stage lead-lag network with time constants $\mathrm{T}_{1}-\mathrm{T}_{4}$ and gain $K_{\mathrm{c}}$ is sufficient to provide adequate damping to the multi-machine power system shown in Figure1.

Optimization techniques based on Lyapunov method and GAs are applied to tune the parameters of a fixed structure lead-lag PSS whose transfer function has the following form (i.e., speed input):

$G_{P S S}(s)=\frac{\Delta u_{i}(s)}{\Delta \omega_{i}(s)}=K_{C i} \cdot\left[\frac{s T_{W i}}{1+s T_{W i}}\right] \cdot\left[\frac{1+s T_{1 i}}{1+s T_{2 i}}\right]\left[\frac{1+s T_{3 i}}{1+s T_{4 i}}\right]$ 
Where, $\mathrm{K}_{\mathrm{Ci}}$ is the stabilizing gain, $\mathrm{T} 1-\mathrm{T}_{4}$ represent suitable time constants of the lead-lag circuit. $T_{W}$ is the washout time constant needed to prevent steady-state offset of the voltage.

The block diagram of the lead-lag PSS on the $\mathrm{i}^{\text {th }}$ machine for dynamic stability studies is shown in Figure 4.

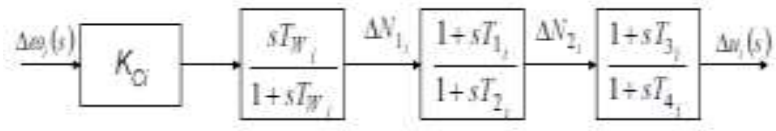

Figure 4. Transfer function block diagram for lead-lag PSS

The PSS parameters to be optimized are the time constants, $\mathrm{T}_{1 i}, \mathrm{~T}_{2 i}, \mathrm{~T}_{3 i}, \mathrm{~T}_{4 i}$ and gain $\mathrm{K}_{C i}$. A washout time constant $\mathrm{T}_{W i}=$ 10 seconds is chosen at all machines in order to ensure that the phase-lead and gain contributed by the washout block for the range of oscillation frequencies normally encountered is negligible [4]. The number of PSS parameters to be optimized is reduced by considering the PSS to comprise two identical cascaded lead-lag networks. Therefore, $\mathrm{T}_{l i}=\mathrm{T}_{3 i}$ and $\mathrm{T}_{2 i}=\mathrm{T}_{4 \mathrm{i}}$. Also, $\mathrm{T}_{2 \mathrm{i}}=\mathrm{T}_{4 \mathrm{i}}=0.05$ seconds is assumed fixed from physical realization considerations [2]. Thus, the optimization problem reduces to determining $\mathrm{T}_{1 \mathrm{i}}$ and $\mathrm{K}_{\mathrm{Ci}}(\mathrm{i}=1, \ldots, \mathrm{n})$ only.

\subsection{Conventional Design of Lead-Lag PSS (CPSS)}

For the sake of clarity, the state-space model of the multi-machine system without PSS is re-stated below:

$$
\frac{d}{d t} \underline{X}(t)=\mathrm{A} \cdot \underline{X}(t)+\mathrm{B} \cdot \underline{U}(t)+\Gamma \cdot \underline{p}(t)
$$

$\mathbf{A}, \mathbf{B}$ and $\boldsymbol{\Gamma}$ are the state, control and perturbation matrices. The associated state, control and perturbation vectors are given below:

$$
\begin{array}{r}
\underline{\mathrm{X}}(\mathrm{t})=\left[\begin{array}{llll}
\Delta \omega_{1}(\mathrm{t}) & \Delta \delta_{1}(\mathrm{t}) & \Delta \mathrm{E}^{\prime} \mathrm{q}_{1}(\mathrm{t}) & \Delta \mathrm{Efd}_{1}(\mathrm{t}) \ldots \\
\Delta \omega_{2}(\mathrm{t}) & \Delta \delta_{2}(\mathrm{t}) & \Delta \mathrm{E}^{\prime} \mathrm{q}_{2}(\mathrm{t}) & \Delta \mathrm{Efd}_{2}(\mathrm{t}) \ldots \\
\Delta \omega_{3}(\mathrm{t}) & \Delta \delta_{3}(\mathrm{t}) & \Delta \mathrm{E}^{\prime} \mathrm{q}_{3}(\mathrm{t}) & \Delta \mathrm{Efd}_{3}(\mathrm{t})
\end{array}\right]^{\mathrm{T}} \\
\underline{\mathrm{U}}(\mathrm{t})=\left[\begin{array}{lll}
\Delta \mathrm{u}_{1}(\mathrm{t}) & \Delta \mathrm{u}_{2}(\mathrm{t}) & \Delta \mathrm{u}_{3}(\mathrm{t})
\end{array}\right]^{\mathrm{T}} \\
\mathrm{p}(\mathrm{t})=\left[\begin{array}{llll}
\Delta \mathrm{T}_{\mathrm{m} 1} & \Delta \mathrm{T}_{\mathrm{m} 2} & \Delta \mathrm{T}_{\mathrm{m} 3}
\end{array}\right]^{\mathrm{T}}
\end{array}
$$

The control vector $\underline{U}(t)$ is a vector of stabilizing signals that represents the PSS output at different machines.

The dynamic equations of the PSS in state-space form, as obtained from the transfer function block-diagram (Figure 4), are given below:

$$
\begin{aligned}
& \frac{d}{d t}\left(\Delta N_{1 i}(t)\right)=K_{C i} \frac{d}{d t}\left(\Delta \omega_{i}\right)-\frac{1}{T_{W i}} \Delta N_{1 i}(t) \\
& \frac{d}{d t}\left(\Delta N_{2 i}(t)\right)=\left[\Delta N_{1 i}(t)-\Delta N_{2 i}(t)+T_{1 i} \frac{d}{d t}\left(\Delta N_{1 i}(t)\right)\right] \cdot \frac{1}{T_{2 i}} \\
& \frac{d}{d t}\left(\Delta u_{i}(t)\right)=\left[\Delta N_{2 i}(t)+T_{3 i} \frac{d}{d t}\left(\Delta N_{1 i}(t)\right)-\Delta u_{i}(t)\right] \cdot \frac{1}{T_{4 i}} \\
& \forall i=1,2,3
\end{aligned}
$$

where $\Delta \mathrm{N}_{1 \mathrm{i}}$ and $\Delta \mathrm{N}_{2 \mathrm{i}}$ are the state-variables associated with each PSS. $\mathrm{T}_{\mathrm{Wi}}$ is the washout time constant, $\mathrm{T}_{1 \mathrm{i}}, \ldots, \mathrm{T} 4 \mathrm{i}$ are the phase-lead time constants and $\mathrm{K}_{\mathrm{Ci}}$ is the stabilizer gain of the PSSs corresponding to each synchronous generator in the power system.
Equations (5) above may be arranged in standard vectormatrix form as shown in (6), which represents the state-space model of the system with PSSs at all machines:

$$
\frac{d}{d t} \underline{X}_{P S S}(t)=\mathrm{C} \cdot \underline{X}(t)+\mathrm{D} \cdot \underline{X}_{P S S}(t)+\Gamma_{1} \cdot \mathrm{p}
$$

where,

$$
\begin{aligned}
& \underline{X}_{P S S}(\mathrm{t})=\left[\Delta \mathrm{N}_{11}(\mathrm{t}) \Delta \mathrm{N}_{21}(\mathrm{t}) \Delta \mathrm{u}_{1}(\mathrm{t}) \ldots\right. \\
& \Delta \mathrm{N}_{12}(\mathrm{t}) \Delta \mathrm{N}_{22}(\mathrm{t}) \Delta \mathrm{u}_{2}(\mathrm{t}) \ldots \\
& \left.\Delta \mathrm{N}_{13}(\mathrm{t}) \quad \Delta \mathrm{N}_{23}(\mathrm{t}) \quad \Delta \mathrm{u}_{3}(\mathrm{t})\right]^{\mathrm{T}}
\end{aligned}
$$

$\mathbf{C}, \mathbf{D}$ and $\boldsymbol{\Gamma}_{\mathbf{1}}$ are the matrices associated with the PSS model with appropriate dimensions.

By defining the following augmented state-vector

$$
\underline{X}_{C}(t)=\left[\underline{X}(t) \quad \underline{X}_{P S S}(t)\right]^{T}
$$

The state-space model of the closed-loop system (composite system) becomes:

$$
\frac{d}{d t} \underline{X}_{C}(t)=\mathrm{A}_{C} \cdot \underline{X}_{C}(t)+\Gamma_{C} \cdot \underline{\mathrm{p}}
$$

where $A_{C}=\left[\begin{array}{cc}A & B_{1} \\ C & D\end{array}\right]$, and $\Gamma_{C}=\left[\begin{array}{ll}\Gamma & \Gamma_{1}\end{array}\right]^{\top}$

$\mathbf{B}_{1}$ in $\mathbf{A} \mathbf{C}$ is a re-defined control matrix, with $\underline{b}_{1}, \underline{b}_{2}, \underline{b}_{3}$ the column vectors of $\mathbf{B}$.

$$
\mathbf{B}_{1}=\left[\begin{array}{lllllllll}
\underline{0} & \underline{0} & \underline{b}_{1} & \underline{0} & \underline{0} & \underline{b}_{2} & \underline{0} & \underline{0} & \underline{b}_{3}
\end{array}\right]
$$

By applying the coordinate transformation in the state-space given in (8), the perturbation term in (7) can be eliminated.

$$
\underline{X}^{\prime}(t)=\underline{X}_{C}(t)-\underline{X}_{C}(\infty)
$$

Hence, (7) reduces to the standard state-variable form:

$$
\frac{d}{d t} \underline{\mathrm{X}}^{\prime}(\mathrm{t})=\mathrm{A}_{C} \cdot \underline{\mathrm{X}}^{\prime}(\mathrm{t})
$$

where $\underline{X}^{\prime}(0)=-\underline{X}(\infty)=-$ Ac. $\Gamma_{C} \cdot \underline{\mathbf{P}}$ is the initial state of $\underline{X}^{\prime}(t)$, which is also the steady-state value of $\underline{X}(t)$.

Conventionally, the PSS tuning methods used for multimachine systems have either used a sequential approach or a simultaneous approach. The choice of a suitable performance index is extremely important for the design of PSS.

In this work, a performance index as given in (10), where $\underline{X}$ is the state vector, and $\mathbf{Q}$ - the weighing matrix - is positive semi-definite and comprises the importance attached to different state-variables in the optimization process, has been used.

$$
J=\int_{0}^{\infty}\left(\mathrm{X}^{T} \cdot \mathrm{Q} \cdot \mathrm{X}\right) d t
$$

The performance index $\mathrm{J}$ can be evaluated using the relation:

$$
\mathrm{J}=\underline{\mathrm{X}}^{\mathrm{T}}(0) \cdot \mathbf{P} \cdot \underline{\mathrm{X}}(0)
$$

Where $\mathrm{X}(0)$ is the initial state of the state-vector, and $\mathbf{P}$ is a positive definite symmetric matrix obtained by solving the following Lyapunov equation:

$$
\mathbf{A}^{\mathrm{T}} \cdot \mathbf{P}+\mathbf{P} \cdot \mathbf{A}=\mathbf{- Q}
$$

where $\mathbf{A}$ is the state matrix of the system. 
By appropriate choice of $\mathbf{Q}$ matrix elements, various penalization weights can be assigned to state variables (which in this case are deviations from steady-state conditions) and a desirable dynamic performance for the system can be achieved.

As described in previously, by an appropriate choice of $\mathbf{Q}$ the performance criterion, and hence the optimal PSS parameters, can be manipulated according to design engineer's requirements. In this work, an Integral of Squared Error (ISE) criterion that seeks to minimize the square of the power angle deviation from its steady-state value $(\boldsymbol{\Delta} \boldsymbol{\delta})$ is chosen. Subsequently, the state variable $\boldsymbol{\Delta} \boldsymbol{\delta}$ is penalized for deviations by being assigned a high weight in the $\mathbf{Q}$ matrix, and the PSS parameters are obtained accordingly.

In the following analysis, the Lyapunov method was applied to multi- machine PSS tuning using the sequential approach.

The weighing matrix $\mathbf{Q}$ is now the sum of the squares of each machine's power angle deviation from their respective steadystate value. Mathematically, this can be written as:

Thus,

$$
\begin{aligned}
\mathbf{Q} & =\operatorname{diag}\left[\begin{array}{llllllllllll}
0 & 1 & 0 & 0 & 0 & 1 & 0 & 0 & 0 & 1 & 0 & 0
\end{array}\right], \text { and } \\
J & =\int_{0}^{\infty} \sum_{i=1}^{3}\left(\Delta \delta_{i}-\Delta \delta_{i S S}\right)^{2} d t
\end{aligned}
$$

In order to obtain the optimal values of $\mathrm{K}_{\mathrm{C}}$ and $\mathrm{T}_{1}$, the following procedure has been used:

1. Choose a set of PSS parameters for which the state matrix of the composite system (including the PSS) is nonsingular.

2. Fix the value of $T_{1}$ and vary $K_{C}$ over a wide range of values and determine the performance index, using (13). It is observed that for a fixed $\mathrm{T}_{1}$, when $\mathrm{K}_{\mathrm{C}}$ increases, the performance index $\mathrm{J}$ decreases continuously attaining a minimum $J_{\min }$ and then start increasing again as $\mathrm{K}_{\mathrm{C}}$ increases further.

3. Carry out Step-2 for various values of $T_{1}$ and determine the minimum $\mathrm{J}_{\min }$.

Using the approach described in the above steps, the parameters of each PSS can be obtained through a sequential optimization approach. Various combinations of tuning sequences were tried out and the best system performance was obtained with MEWA (Gen-1)-AWASH2 (Gen-2)-KOKA (Gen-3) sequence.

\subsection{GA Based Design of Lead-Lag PSS (GA-PSS)}

The proposed Lyapunov method based genetic algorithm is initiated by generating randomly an initial population of binary coded individuals, where each individual represents a possible solution for the PSS parameters.

A basic requirement for obtaining a feasible solution to the Lyapunov equation is that the state-matrix A should be stable. Fulfillment of this condition is ensured by stability screening. The entire population of individuals in each generation is screened in order to ensure that only those individuals (each of them representing a PSS parameter set) that provide a stable system over the whole operating domain D, are allowed to proceed further in the optimization process.

This also brings about significant reduction in the computational burden. Individuals resulting in unstable systems for an operating point within the domain D ("bad individuals") are assigned a very high value of $\mathrm{J}_{\mathrm{AVG}}$, where $\mathrm{J}_{\mathrm{AVG}}$ is the mean value of performance indices over the $\mathrm{N}_{\mathrm{op}}$ points of the operating domain $\mathrm{D}$, and given by (14). The bad individuals are gradually phased out from the population within a few generations.

$$
J_{A V G}=\frac{1}{N_{o p}} \sum_{P, Q}\left(\int_{0}^{\infty}\left(\mathrm{X}^{T} \cdot \mathrm{Q} \cdot \mathrm{X}\right) d t\right)
$$

$\forall P, Q \in D$

Every individual (chromosome) of the current population is evaluated for $\mathrm{J}_{\mathrm{AVG}}$ and a basis for the biased selection process is then established. To avoid premature convergence and speeding up of the search when the convergence is approached, the objective values obtained for each individual are mapped into fitness values through a ranking process.

The rank-based fitness assignment overcomes the scaling problems of the proportional fitness assignment. The individuals will be ranked in the population in descending order of their fitness with respect to the problem domain. The higher the individual's fitness is, the higher is its chance to pass-on genetic information to successive generations.

The next generation will be populated with offspring, obtained from selected parents. The selection is a process used to determine the number of trials for one particular individual used in reproduction. The selection process uses the stochastic universal sampling method, a single-phase sampling algorithm with minimum spread, zero bias and time complexity in the order of the number of individuals (NIND).

Recombination of the selected individuals is carried out with pairs of individuals from the current population using a multipoint crossover process having a certain probability [19]. The individuals in the pairs will exchange genetic information with each other, thereby creating two new individuals, the offspring. After that, each individual in the population will be mutated with a given probability, through a random process of replacing one allele of a gene with another to produce a new genetic structure.

The GA employed in this study uses an elitist strategy in which the offspring is created with a prespecified generation gap and reinserted in the old population by replacing the least fit predecessors. Most fit individuals are allowed to propagate through successive generations and only a better individual may replace them [17].

The GA stops when a pre-defined maximum number of generations is achieved or when the value returned by the objective function, being below a threshold, remains constant for a number of iterations.

\subsection{PSS Parameters}

The parameters of the CPSS, the GA-PSSs are listed in Tables $1-2$, respectively. It can be seen that for both CPSS and GAPSSs, $\mathrm{T}_{1}=\mathrm{T}_{3}, \mathrm{~T}_{2}=\mathrm{T}_{4}=0.05$ and $\mathrm{T}_{W}=10$ seconds. This is in accordance with the general practice, and thus the optimal PSS parameter obtained from the optimization solutions are $\mathrm{T}_{1}$ and $\mathrm{K}_{\mathrm{C}}$ for each PSS at each generator of the power system considered in this paper. 
Table 1. Parameters of the CPSS

\begin{tabular}{llll}
\hline Parameters & MEWA & AWASH2 & KOKA \\
& $($ Gen-1 $)$ & $($ Gen-2) & $($ Gen-3) \\
\hline $\mathrm{K}_{\mathrm{C}}$ & $\mathbf{2 . 9 7}$ & $\mathbf{1 . 0 9}$ & $\mathbf{0 . 7 3}$ \\
$\mathrm{T}_{W}(\mathrm{sec})$ & $\mathbf{1 0}$ & $\mathbf{1 0}$ & $\mathbf{1 0}$ \\
$\mathrm{T}_{1}(\mathrm{sec})$ & $\mathbf{0 . 1 0}$ & $\mathbf{0 . 1 4}$ & $\mathbf{0 . 2 6}$ \\
$\mathrm{T}_{2}(\mathrm{sec})$ & $\mathbf{0 . 0 5}$ & $\mathbf{0 . 0 5}$ & $\mathbf{0 . 0 5}$ \\
$\mathrm{T}_{3}(\mathrm{sec})$ & $\mathbf{0 . 1 0}$ & $\mathbf{0 . 1 4}$ & $\mathbf{0 . 2 6}$ \\
$\mathrm{T}_{4}(\mathrm{sec})$ & $\mathbf{0 . 0 5}$ & $\mathbf{0 . 0 5}$ & $\mathbf{0 . 0 5}$ \\
\hline
\end{tabular}

Table 2. Parameters of the GA-PSS

\begin{tabular}{llll} 
& MEWA & A WASH2 & KOKA \\
Parameters & $($ Gen-1 $)$ & $($ Gen-2 $)$ & $($ Gen-3) \\
\hline $\mathrm{K}_{\mathrm{C}}$ & $\mathbf{3 . 0}$ & $\mathbf{1 . 2}$ & $\mathbf{0 . 8}$ \\
$\mathrm{T}_{W}(\mathrm{sec})$ & $\mathbf{1 0}$ & $\mathbf{1 0}$ & $\mathbf{1 0}$ \\
$\mathrm{T}_{1}(\mathrm{sec})$ & $\mathbf{0 . 1 2}$ & $\mathbf{0 . 1 8}$ & $\mathbf{0 . 2 3}$ \\
$\mathrm{T}_{2}(\mathrm{sec})$ & $\mathbf{0 . 0 5}$ & $\mathbf{0 . 0 5}$ & $\mathbf{0 . 0 5}$ \\
$\mathrm{T}_{3}(\mathrm{sec})$ & $\mathbf{0 . 1 2}$ & $\mathbf{0 . 1 8}$ & $\mathbf{0 . 2 3}$ \\
$\mathrm{T}_{4}(\mathrm{sec})$ & $\mathbf{0 . 0 5}$ & $\mathbf{0 . 0 5}$ & $\mathbf{0 . 0 5}$ \\
\hline
\end{tabular}

\section{CASE STUDY AND SIMULATION RESULTS}

In order to test the robustness of the PSSs, three different operating conditions were considered. The different operating conditions (represented by light, nominal and heavy loading) are given in Table 3. It might be noted that these load conditions at buses 5, 7 and 8, are same as those load variations expected in the system, except that the corresponding generation levels are obtained here using an OPF simulation with "minimizing losses" as objective.

The simulation results are presented in two ways, first the system eigenvalues with and without PSSs are presented, and in the second the system dynamic responses are plotted for both the GA based PSS and the ISE technique based PSS. The dynamic responses are plotted for rotor angle deviations of all the system generators following a $1 \%$ step change in mechanical torque input on MEWA (Gen-1) generator.
Table 3. Three different loading conditions for examining the performance of the PSSs

\begin{tabular}{|l|l|l|l|l|l|l|}
\hline \multirow{2}{*}{ Cases } & \multicolumn{2}{|l|}{ MEWA } & \multicolumn{2}{l|}{ AWASH2 } & \multicolumn{2}{l|}{ KOKA } \\
& \multicolumn{2}{|l|}{ (Gen-1) } & \multicolumn{2}{l|}{ (Gen-2) } & \multicolumn{2}{l|}{ (Gen-3) } \\
\cline { 2 - 7 } & $\mathrm{P}_{\mathrm{e}}$ & $\mathrm{Q}_{\mathrm{e}}$ & $\mathrm{P}_{\mathrm{e}}$ & $\mathrm{Q}_{\mathrm{e}}$ & $\mathrm{P}_{\mathrm{e}}$ & $\mathrm{Q}_{\mathrm{e}}$ \\
\hline Light & 0.17 & 0.03 & 0.11 & -0.11 & 0.90 & -0.12 \\
\hline Nominal & 0.35 & 0.1027 & 0.13 & 0.07 & 0.10 & -0.1081 \\
\hline Heavy & 0.468 & 0.87 & 0.18 & 0.60 & 0.144 & 0.546 \\
\hline
\end{tabular}

All the values are given in per-unit

\subsection{Eigenvalue Analysis}

The eigenvalues of the open-loop system without PSS and the closed-loop system equipped with the CPSSs, and the GAPSSs are listed in Table 4. It can be seen from the open-loop eigenvalues listed in Table 4, that there are two eigenvalues which lie in the right half of the S-plane (has positive real part). This shows that there are electromechanical oscillations persistent in the system and the system remains dynamically unstable without PSS

Columns 2 and 3 in Table 4 show the eigenvalues obtained with the CPSSs, and the GA-PSSs, respectively. It can be seen that for all the loading cases, the CPSSs provide acceptable damping ratio. The GA-PSSs provide "consistent" damping ratio across the range of operating conditions considered. Furthermore, all the eigenvalues obtained with the CPSSs, and the GA-PSSs lie to the left of the complex S-plane (have negative real parts). This shows that the electromechanical oscillations in the system are damped out and the system has gained its dynamic stability.

Table 4. Eigenvalues of the open-loop system (without PSS), closed-loop system with CPSS, and GA-PSS

\begin{tabular}{|c|c|c|}
\hline $\begin{array}{c}\text { System } \\
\text { Eigenvalues } \\
\text { Without PSS }\end{array}$ & $\begin{array}{c}\text { System } \\
\text { Eigenvalues } \\
\text { With CPSS }\end{array}$ & $\begin{array}{c}\text { System } \\
\text { Eigenvalues } \\
\text { With GA-PSS }\end{array}$ \\
\hline$-5.32+\mathrm{j} 22.9$ & $-2.2526 \pm \mathrm{j} 21.1140$ & $-2.8935 \pm \mathrm{j} 22.4227$ \\
$-5.32-\mathrm{j} 22.9$ & $-2.5951 \pm \mathrm{j} 17.7718$ & $-1.6304 \pm \mathrm{j} 16.8463$ \\
$-5.35+\mathrm{j} 19.4$ & $-4.4733 \pm \mathrm{j} 16.1442$ & $-4.8087 \pm \mathrm{j} 16.7140$ \\
$-5.35-\mathrm{j} 19.4$ & $-0.6712 \pm \mathrm{j} 9.2975$ & $-0.3606 \pm \mathrm{j} 9.0937$ \\
$-0.00801+\mathrm{j} 16.0$ & $-0.2654 \pm \mathrm{j} 5.9188$ & $-0.8613 \pm \mathrm{j} 6.0866$ \\
$-0.00801-\mathrm{j} 16.0$ & $-4.7354 \pm \mathrm{j} 9.7216$ & $-3.9211 \pm \mathrm{j} 9.4440$ \\
$-5.10+\mathrm{j} 10.8$ & $-18.1360 \pm \mathrm{j} 0.0806$ & $-20.3341 \pm \mathrm{j} 1.5866$ \\
$-5.10-\mathrm{j} 10.8$ & -23.3570 & -25.2256 \\
$0.0128+\mathrm{j} 5.39$ & -22.6372 & -24.7753 \\
$0.0128-\mathrm{j} 5.39$ & -22.0002 & -15.9589 \\
$-0.0163+\mathrm{j} 8.44$ & -17.6208 & -16.2935 \\
$-0.0163-\mathrm{j} 8.44$ & -0.1001 & -0.1004 \\
& -0.1000 & -0.1000 \\
& -0.1001 & -0.1000 \\
\hline
\end{tabular}


From an eigenvalue point of view, the performance of GAPSSs is better than that of the CPSSs over the range of operating conditions considered. For nominal and heavy loading cases, the damping ratio provided by the GA-PSS is greater than damping ratio provided by the CPSS.

\subsection{Time Domain Simulations}

Non-linear time domain simulations have been performed to evaluate the performances of the system under various loading conditions discussed in the above sections.

For all the following simulations, the dynamic responses are plotted for rotor angle and speed deviations of the system generators following a $1 \%$ step change in mechanical torque input on MEWA (Gen-1) generator. The responses are plotted for both the GA based PSS (GA-PSS) and the ISE technique based PSS (CPSS).

Figure 5 and Figure 6, respectively, show the time response of rotor angle and angular speed deviations of all the machines of the system without PSS, when a $1 \%$ step perturbation in mechanical torque input occurs at the shaft of MEWA (Gen-1) generator.

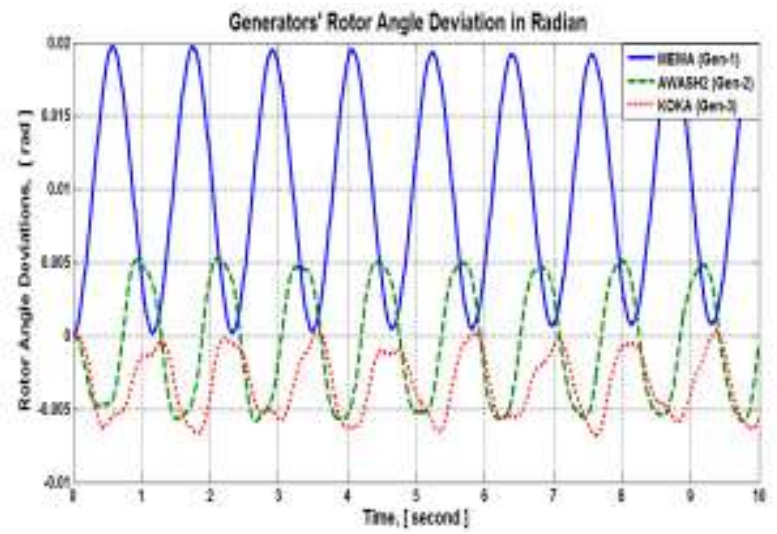

Figure 5. Rotor angle deviations without PSS

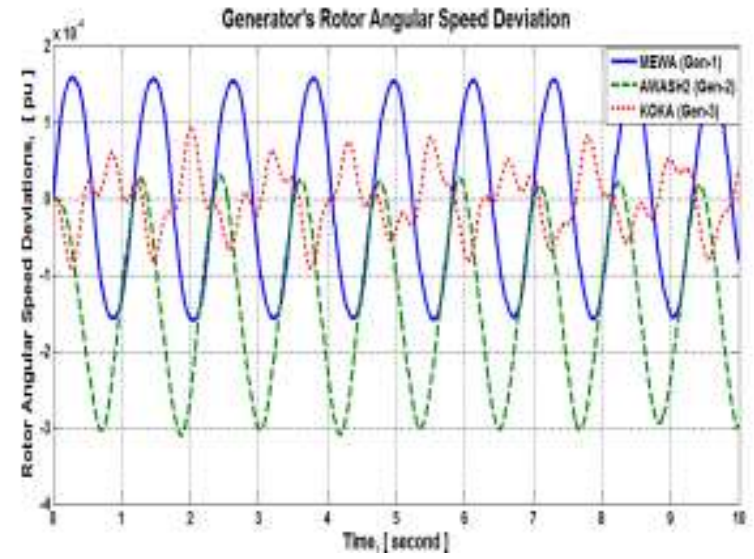

Figure 6. Rotor angular speed deviations without PSS

As has been demonstrated by Figures 5 and 6, the system is unstable under small perturbations and requires additional stabilizing control signals from PSSs.
Figures 7 and 8 show the plots for the nominal operating load condition. The GA based PSS has a lower peak off-shoot and smaller oscillations and an overall better damped response.

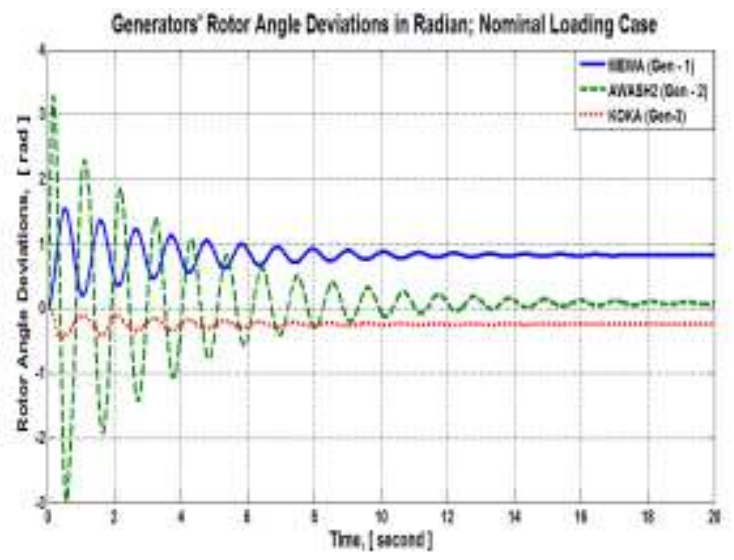

Figure 7. Rotor angle deviations with CPSS: nominal load condition

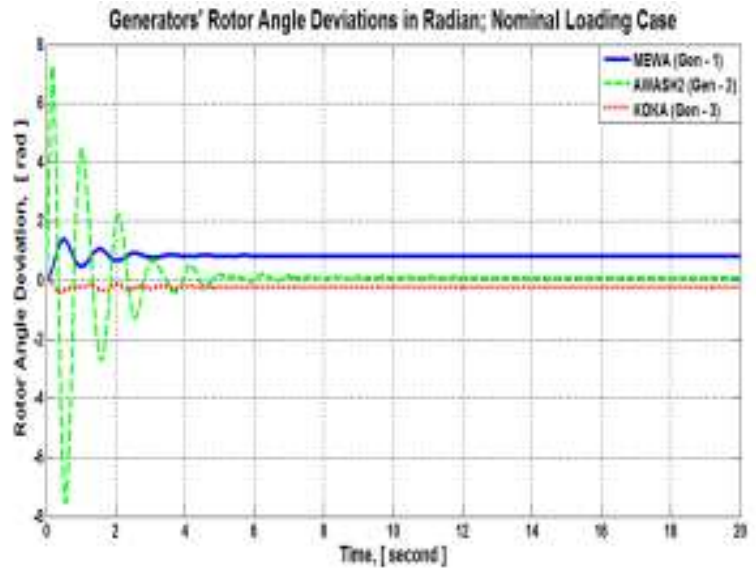

Figure 8. Rotor angle deviations with GA-PSS: nominal load condition

Figures 9 and 10 show the plots for the heavy operating load condition. It is evident that the GA based PSS performs distinctly better as compared to the ISE technique based PSS (CPSS).

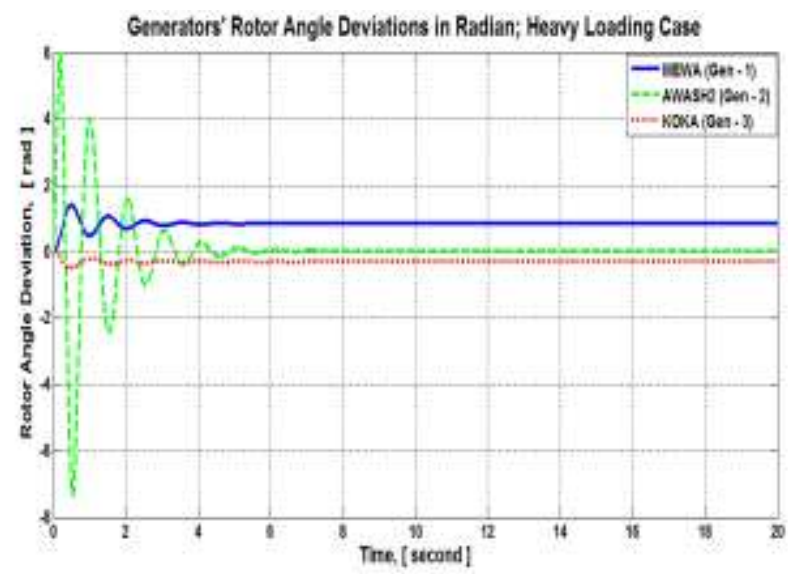

Figure 9. Rotor angle deviations with CPSS: heavy load condition 


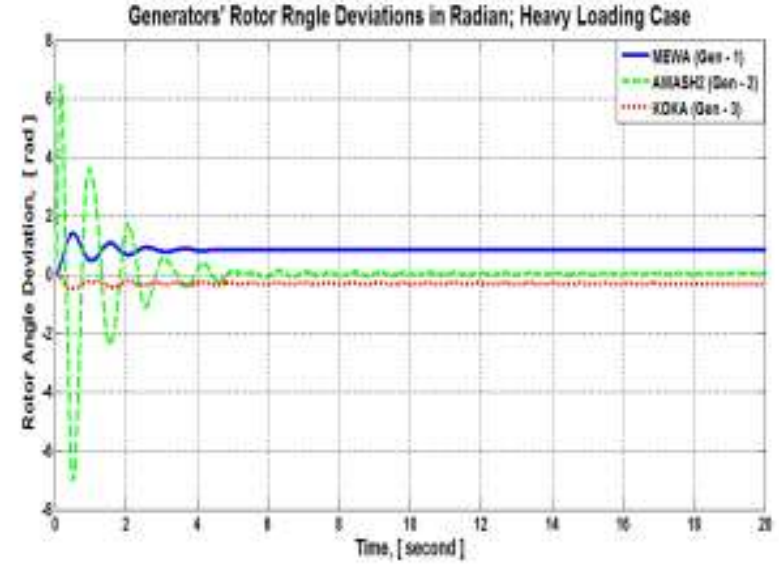

Figure 10. Rotor angle deviations with GA-PSS:

heavy load condition

Figures 11 and 12 show the plots for the light operating load condition. In this case, the GA based PSS and the ISE technique based PSS both do provide satisfactory responses.

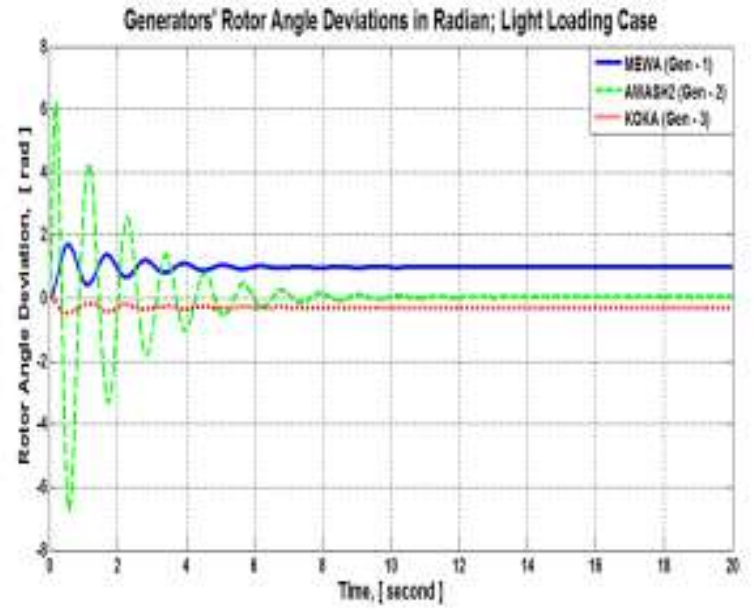

Figure 11. Rotor angle deviations with CPSS:

light load condition

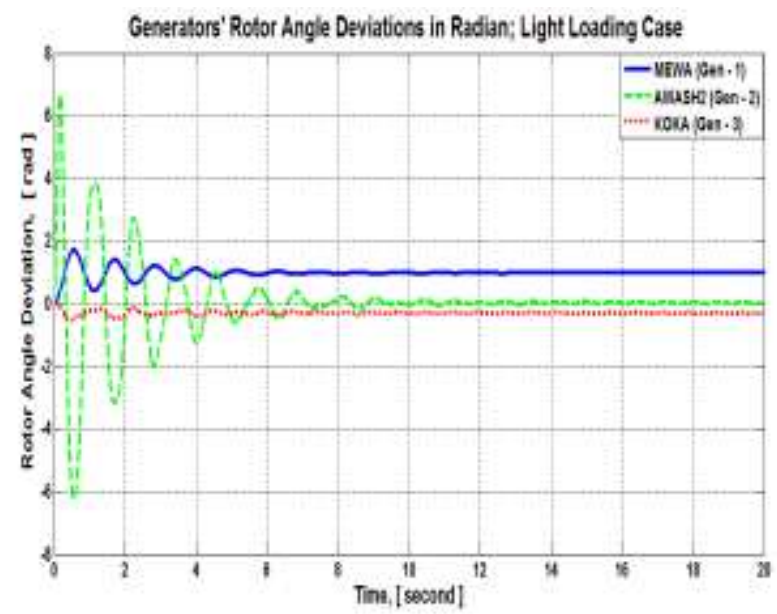

Figure 12. Rotor angle deviations with GA-PSS:

light load condition

\section{CONCLUSIONS}

Power system stabilizer design for multimachine power system using GA has been presented in this paper. The issue of tuning the parameters of the PSSs has been converted into an optimization problem which is solved via GA. Without PSSs, it has been demonstrated that the operation of the multimachine power system considered in this paper is dynamically unstable (has poorly damped electromechanical oscillations). The low frequency electromechanical oscillations have been completely damped out and the power system examined, in this research paper, has become dynamically stable with the design and integration of PSSs.

Moreover, eigenvalue analysis show that the GA-PSSs perform better than the CPSSs and give adequate and consistent damping for all the three-cases considered. Matlab nonlinear time domain simulations are presented to confirm the eigenvalue analysis results. The main advantage of GA is that it is simple, easy to use and has few genetic operators, and robust to solve the fitness function used to tune the optimal PSS parameters.

\section{REFERENCES}

[1] B.E. Eliasson and D.J. Hill, "Damping Structure and Sensitivity in the Nordel Power System", IEEE Transactions on Power Systems, Vol. 7, No. 1, February 1992, pp. 97-105.

[2] F.P. DeMello and C. Concordia, "Concepts of synchronous machines stability as affected by excitation control," IEEE Trans. on Power Apparatus and Systems, Vol. PAS-88, April 1969, pp. 316-329.

[3] W.G. Heffron, R.A. Phillips, "Effect of modern amplidyne voltage regulator on under-excited operation of large turbine generators," IEEE Transactions on Power Apparatus and Systems, Vol. PAS-71, August 1952, pp. 692-697.

[4] P. Kundur, M. Klein, G.J. Rogers and M.S. Zywno, "Application of power system stabilizers for enhancement of overall system stability," IEEE Transactions on Power Systems, Vol. 4, May 1989, pp. 614-626.

[5] Y.L. Abdel-Magid, M.A. Abido, S. Al-Baiyat and A.H. Mantawy, "Simultaneous stabilization of multimachine power systems via genetic algorithms," IEEE Transactions on Power Systems, Vol. 14, November 1999, pp. 1428-1439.

[6] Y.L Abdel-Magid, M.A. Abido, "Optimal multiobjective design of robust power system stabilizers using genetic algorithms," IEEE Transactions on Power Systems, Vol. 18, No. 3 , August 2003, pp.1125-1132

[7] P. Zhang and A.H. Coonick, "Coordinated Synthesis of PSS Parameters in Multi-Machine Power Systems Using the Method of Inequalities Applied to Genetic Algorithms," IEEE Transactions on Power Systems, Vol. 15, No. 2, May 2000, pp. 811-816.

[8] A.L.B. Do Bomfim, G.N. Taranto, D.M. Falcao, "Simultaneous tuning of power system damping controllers using genetic algorithms," IEEE Transactions on Power Systems, Vol. 15, No. 1, February 2000, pp. 163-169.

[9] P. Kundur,"A Course on Power Stability and Control," ABB T\&D University, Ludvika, Sweden, April 2000. 
[10] Yao-Nan Yu, Electric Power System Dynamics, Academic Press 1983.

[11] D.E. Goldberg, Genetic Algorithms in Search, Optimization and Machine Learning, Addison-Wesley Publishing Company Inc., January 1989.

[12] "Western Electricity Coordinating Council Policy Statement on Power System Stabilizers" [Online]. Available: http://www.wecc.biz

[13] "General Transmission System Design Requirements for the Interconnection of New Generators (Resources) to the NEPOOL System" [Online]. Available: ttp://www.iso-ne.com

[14] B.E. Eliasson, "Damping of Power Oscillations in Large Power Systems", PhD thesis TFRT-1032, Department of Automatic Control, Lund University of Technology, Lund, Sweden, May 1990.

[15] Ethiopian national electric power grid, 2012.

[16] K FOLLY, "Power System Stabilizer Design for Multimachine Power System Using Population-Based Incremental Learning", Power Plants and Power Systems Control, 2006, 2007.

[17] K. Bhattacharya, "Lyapunov's method based genetic algorithm for multi-machine PSS tuning", 2002 IEEE Power Engineering Society Winter Meeting Conference Proceedings (Cat No 02CH37309 PESW-02), 2002.

[18] Bhattacharya, K., "Tuning of power system stabilizers in multi-machine systems using ISE technique", Electric Power Systems Research, 199806.

[19] Andreoiu, A., and K. Bhattacharya, "Robust tuning of power system stabilisers using a Lyapunov method based genetic algorithm", IEEE Proceedings - Generation, Transmission and Distribution, 2002.

[20] www.elkraft.chalmers.se

[21] www.ijmer.com

[22] www.elteknik.chalmers.se

[23] waste.org

[24] Shayeghi, H., and A. Ghasemi, "A multi objective vector evaluated improved honey bee mating optimization for optimal and robust design of power system stabilizers", International Journal of Electrical Power \& Energy Systems, 2014.

[25] He Ren-mu, "Genetic algorithm based design of power system stabilizers", 2004 IEEE International Conference on Electric Utility Deregulation Restructuring and Power Technologies Proceedings, 2004.

[26] Shayeghi, H., "Multi-machine power system stabilizers design using chaotic optimization algorithm", Energy Conversion and Management, 201007.

[27] www.edi-info.ir

[28] Aalok Dubey, "Simultaneous Stabilization of Multimachine Power System Using Genetic Algorithm", Proceedings of the 41st International Universities Power Engineering Conference, September 2006.

[29] Liu, N., J. Chen, L. Zhu, J. Zhang, and Y. He, “A Key Management Scheme for Secure Communications of Advanced Metering Infrastructure in Smart Grid", IEEE Transactions on Industrial Electronics, 2012.

[30] www.docstoc.com
[31] Adrian Andreoiu, "On Power System Stabilizers: Genetic Algorithm Based Tuning and Economic Worth as Ancillary Services", $\mathrm{PhD}$ thesis, Department of Electric Power Engineering, Chalmers University of Technology, Goteborg, Sweden, 2004. 